\title{
Toward Sensor Intelligence in MEMS Technology
}

\author{
Kazusuke Maenaka \\ Graduate School of Engineering, University of Hyogo \\ 2167, Shosha, Himeji, Hyogo 671-2280, Japan
}

(Received December 17, 2017; accepted January 15, 2018)

Keywords: integrated sensor, intelligent seonsor, smart sensor, combo sensor, sensor network

In the past 30 years, remarkable progress has been made in the field of MEMS sensors. The journal Sensors and Materials has reached its 30th year and has been documenting the progress of microsensor technology. Also, I have been working on MEMS sensors for around 30 years, and watching the progress of the MEMS field. Recently, almost all physical sensors have been realized by MEMS technology and many dreams and expectations in the old days have become real devices. In this paper, I will focus on intelligence or smartness of sensors as the subject with some historical aspects.

\section{Introduction}

Before proceeding to the main topic, I would like to introduce myself. My academic background is on information technology and related hardware. I graduated from the Department of Computer Science and Engineering of Toyohashi University of Technology in 1984. As an M.E. student, my research area was on image recognition systems including hardware. After graduation, as a research assistant, I joined the Laboratory of Electron Devices in the Department of Electrical and Electronic Engineering of Toyohashi University of Technology. The head of the laboratory was Prof. Tetsuro Nakamura, who was one of the past editors-in-chief of Sensors and Materials. I started research on sensors and MEMS technology under Prof. Nakamura and with Prof. Makoto Ishida (current Editor-in-Chief of the journal), who was associate professor at that time in the laboratory. At first, I worked on magnetic sensors and their integration, particularly on multi-dimensional integrated and intelligent magnetic sensors. In those days, almost all sensors were scalar sensors even if the sensing target is a vector quantity, and multi-dimensional sensing was an interesting subject. Until now, although my circumstances have changed, I have been continuously working on MEMS sensors, their integration and related technology. In this paper, I would like to describe briefly MEMS history on the basis of my own experiences.

*Corresponding author: e-mail: maenaka@eng.u-hyogo.ac.jp http://dx.doi.org/10.18494/SAM.2018.1856 


\section{Development of MEMS Technology}

\subsection{Based on Integrated Circuit Technology}

In 1980s, many microdevices, including MEMS sensors, were presented along with the rapid development of integrated circuit technologies especially microprocessors. Fine patterning, thin-film deposition, oxidation, impurity diffusion, and so forth, which are basic technologies of integrated circuits, became the essential technologies of microdevices. Additionally, anisotropic wet etching that forms a precise diaphragm or a movable mass was also a powerful technique for microdevice fabrication. ${ }^{(1)}$ Some microsensor devices such as pressure sensors were presented at that time.

\subsection{Integration of sensors with bipolar circuit technology}

In order to realize intelligent sensors, an integration of sensors with circuits attracted attention in the 1980s. Because a sensor output is usually very small and unstable, an on-chip amplifier for a detected signal is the minimum requirement for integrated sensors. Moreover, not only an amplifier but highly functional circuits for signal processing with analog and digital circuitry were proposed. ${ }^{(2,3)}$ It might be linked with the rapid progress of digital microprocessors. However, at that time, metal-oxide-semiconductor (MOS) analog technology was not well developed, and the integration of sensors with analog and digital processors in one chip was a premature idea (of course, today, MOS analog technology has sufficiently progressed for the sensor interface ${ }^{(4)}$ ). Instead of a digital MOS circuit, bipolar analog processing was adopted for sensors and some of them have been reported and delivered to the market. Figure 1 shows the acceleration sensors with an amplifier on the chip ${ }^{(5)}$ and Fig. 2 shows a pressure
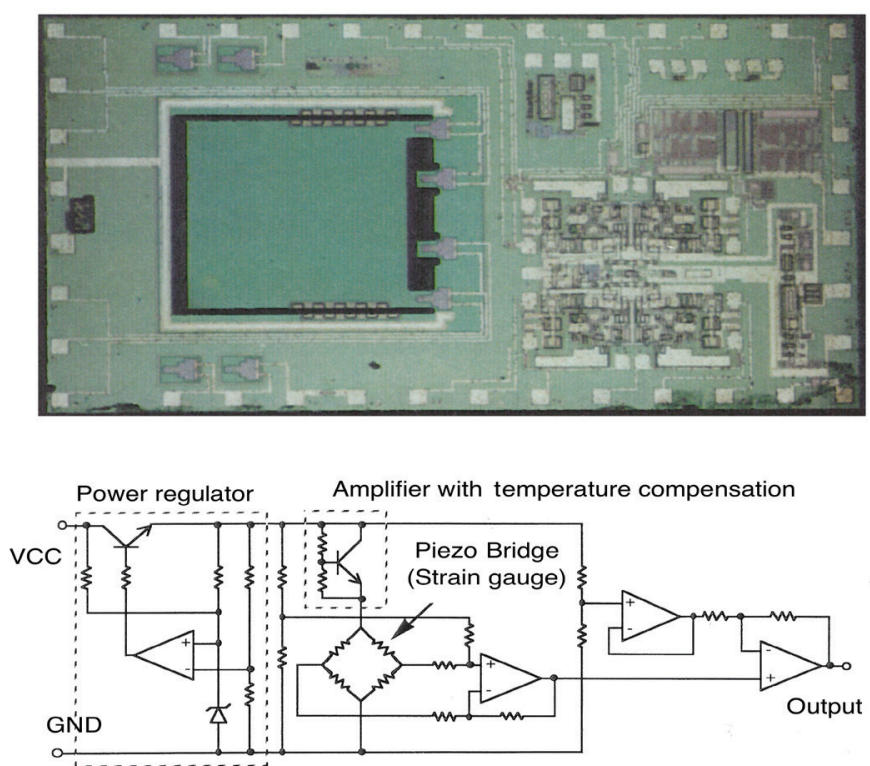

Fig. 1. (Color online) Early integrated acceleration sensor-chip photo and internal circuits. 


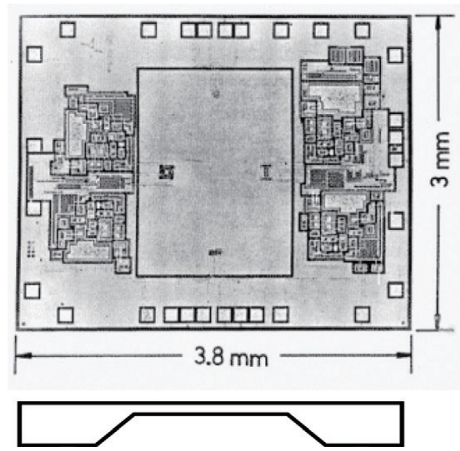

(a)

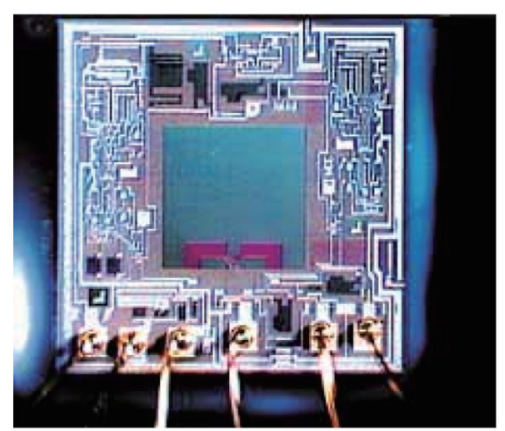

(b)

Fig. 2. (Color online) (a) Early integrated pressure sensor ${ }^{(6)}$ and (b) commercialized one. ${ }^{(7)}$

sensor with an amplifier and compensation circuits. ${ }^{(6,7)}$ Not only simple circuits, but sensors with some signal processing circuits were proposed. Figures 3-5 are examples of such devices presented by our group. These devices incorporate magnetic sensors with some analog calculation circuit in the sensor chip. The device shown in Fig. 3 includes a three-dimensional magnetic sensor and a bipolar absolute calculation circuit $\left(=\sqrt{B_{x}^{2}+B_{y}^{2}+B_{z}^{2}}\right)$ in one chip. ${ }^{(8)}$ This enables omnidirectional measurement of the magnetic field. The analog calculation is performed by the translinear scheme. In Fig. 4, a two-dimensional magnetic sensor is integrated with absolute value $\left(=\sqrt{B_{x}^{2}+B_{y}^{2}}\right)$ and incline angle $\left(=\tan ^{-1}\left(\frac{B_{y}}{B_{x}}\right)\right)$ calculation circuits. ${ }^{(9)}$ This can be used as a magnetic compass. The device in Fig. 5, named magneto-operational amplifier (MOP), has the functionality of an operational amplifier, where some feedback elements define the mathematical operation for a magnetic field such as arbitrary amplification, current output, differentiation and integration operation for a magnetic field in the time domain, magneticfield-controlled oscillation, and switching with hysteresis. ${ }^{(10)}$ Reference 10 shows 10 types of magnetic operation circuits and measured results using an MOP with various feedback elements.

\subsection{Inertial sensors}

Inertial sensors, that is, acceleration sensors and gyroscopes, are worthy of MEMS devices because they essentially have movable mechanical parts. As for acceleration sensors, in the early stage, it was said that the device was suitable for the air bag system of an automobile (detection of collision) and the development was driven in a positive manner. Thus, acceleration sensors were well studied in the early stage of MEMS development. In particular, ADXL50 ${ }^{(11)}$ from Analog Devices Inc., released in 1993, and the following ADXL series were elegant devices in which a sensing element formed by poly-Si and (a bipolar-based) Bi-CMOS analog interface are integrated together in one monolithic chip.

As for a gyroscope, because angular rate (angular velocity) detection is very difficult owing to the very small output signal of a small device and it requires constant vibration of the mass (called reference vibration) in order to generate Coriolis force, the development of gyroscopes 

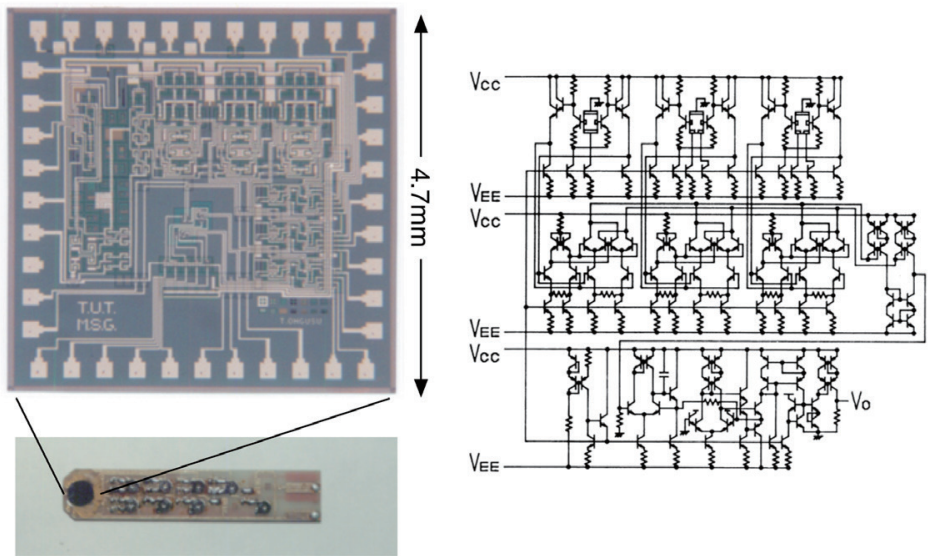

Fig. 3. (Color online) Omnidirectional magnetic sensor - 3D magnetic sensor is integrated with bipolar absolute calculation circuitry.

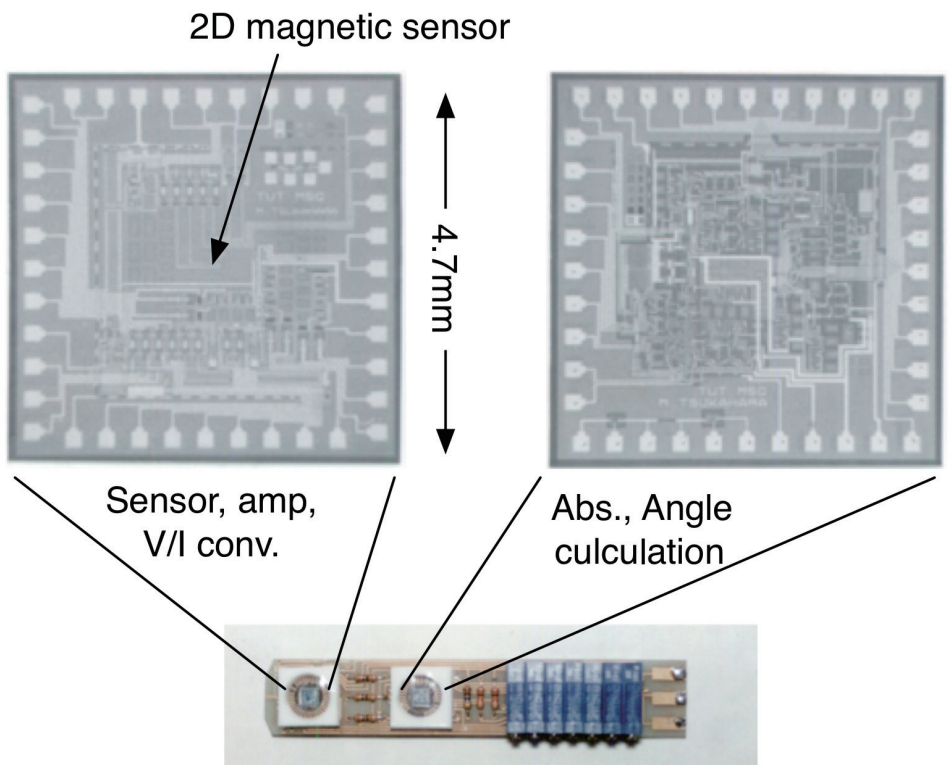

Fig. 4. (Color online) Integrated magnetic sensor with outputs of absolute value and direction of 2D magnetic field.
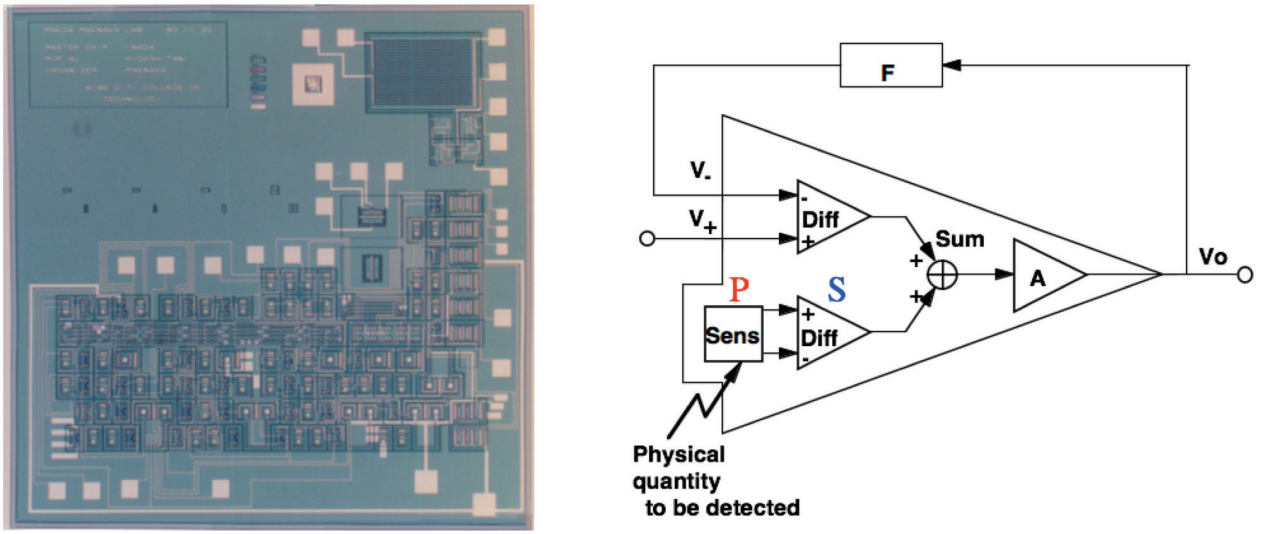

Fig. 5. (Color online) Magneto-operational amplifier: by arranging the feedback scheme, many magnetic operations can be performed. 
did not proceed easily. Beginning in 1990, our group presented a simple gyroscope that has a single cantilever as a vibrating mass, as shown in Fig. $6^{(12)}$ To secure a large mass, bulk micromachining with anisotropic wet etching was adopted. Owing to crystal anisotropy in the wet etching, the total shape of the device is not regular or symmetric. However, this work demonstrated that the silicon device could detect an angular rate with even a simple structure. For commercialization, Analog Devices Inc. delivered a monolithic gyroscope ${ }^{(13)}$ (ADXRS series) using the same technology as the ADXL series in 2002. This is world's first monolithic integrated gyroscope. These devices acted as an initiator of the development of MEMS gyroscopes, and following them, many inertial sensing devices from other companies have been introduced in the market.

\subsection{Bosch process}

One of the epoch-making technologies is the Bosch process in $1995,{ }^{(14)}$ which is a special dry etching technology with extremely deep and vertical etching capability. This technology markedly widened the flexibility of the structure design. Figure 7 shows some etching results using the Bosch process. This process enables arbitral surface design for sensor devices with high accuracy and it is especially useful for inertial sensors, which have special spring and mass structures.
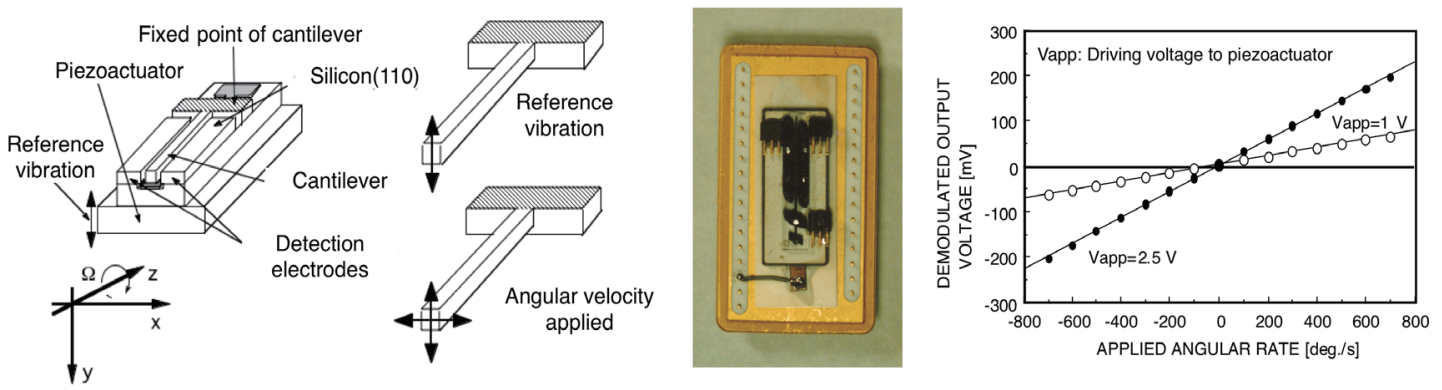

Fig. 6. (Color online) Simple cantilever-type gyroscope in the early days.
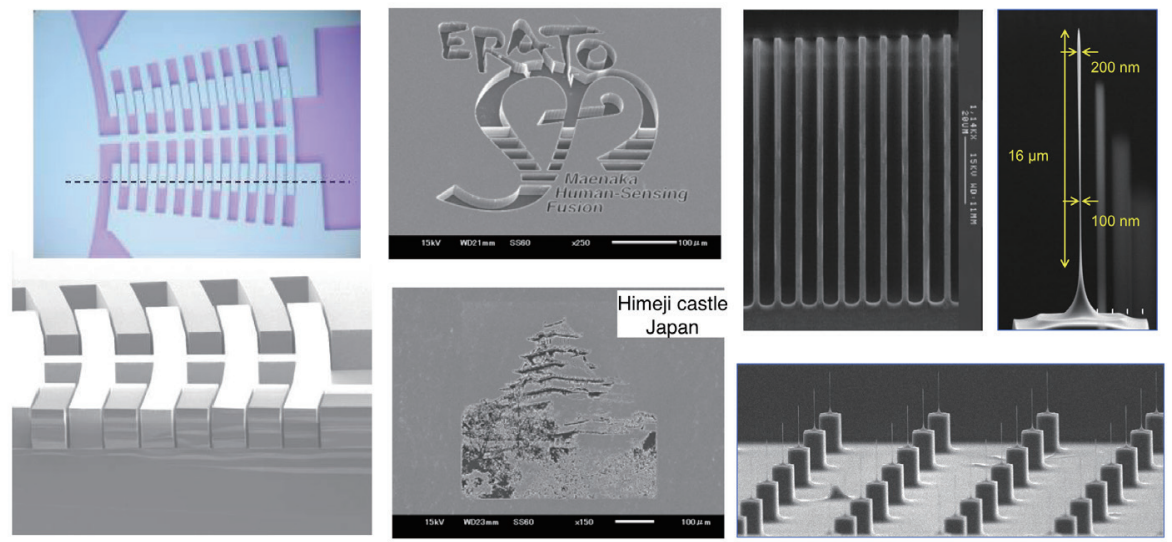

Fig. 7. (Color online) Examples of deep etching by Bosch process-with the use of sacrificial etching, very narrow needles can be obtained. ${ }^{(24)}$ 


\subsection{Monolithic/hybrid and combo sensors}

In the old days, I insisted that intelligent/smart sensors should have a "monolithic" form, in which a sensor is incorporated with a peripheral circuitry in one monolithic die, as shown in Figs. 1-5. Also, the world's first integrated inertial sensors, the ADXL and ADXRS series, were monolithic devices. However, in the current market, almost all devices have the hybrid scheme, in which sensor dies and integrated circuit dies are arranged horizontally and/or vertically and bonding wires connect between dies and dies, and between dies and the package stem. In the current technological stage, the hybrid scheme should have more merit than the monolithic one from the viewpoint of cost. Particularly for combo sensors, which combine some types of sensors (for example, a 3D acceleration sensor, a 3D gyro sensor, and a 3D magnetic sensor) in one package, the hybrid scheme is a simpler building technology for realization because previously developed devices can be used without further development. By this method, the current MEMS sensor not only has a sensor structure but also a highly functional digital circuit, such as digital signal compensation, variable gain/frequency characteristics, I2C/SPI digital interface capability, programmable interrupt for certain input signals, and so on in a small package. Examples of current MEMS sensors are shown in Fig. 8.

Despite the current situation in the market, I would like to claim that monolithic integration is the ultimate technology for future smart sensors. Although it is old fashioned (presented in 2001: combined circuitry was designed by bipolar analog technology), an example of an early monolithic combo sensor with peripheral circuitry is shown in Fig. 9. ${ }^{(15)}$ This work may be the first stage of combo sensors. Today, to adapt to the future Internet of Things (IoT) and Trillion Sensors Universe, some projects related to "smart and monolithic" combo sensors have been started throughout the world.

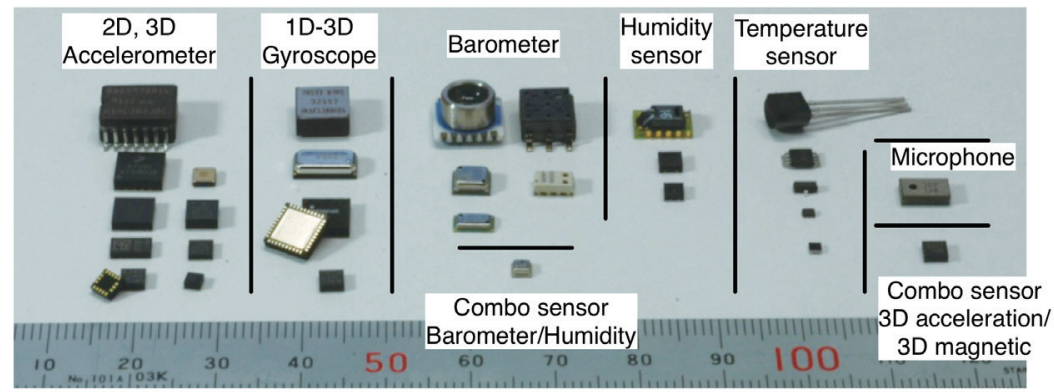

Fig. 8. (Color online) Current MEMS sensors in the market. Devices in the upper part of the picture are old devices.

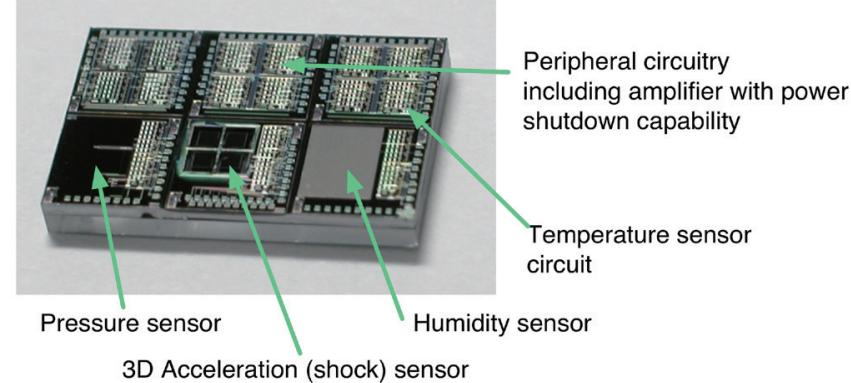

Fig. 9. (Color online) Early combo sensor with bipolar circuitry. 


\section{Network and Sensors}

We started a study of the connection between network and multiple sensors in 2001. Figure 10 shows the appearance of an example system. In 2001, there is no wireless LAN for which a common user can use easily and no one-chip processor with low power. Thus, the system has a wired LAN, single-board Linux system, A/D converter IC, and 5 AA batteries as well as a sensor module (shown in Fig. 9). The case size is large at $200 \times 120 \times 25 \mathrm{~mm}^{3}$. Because the system had Web server capability, networked clients at a distant place from the sensor system could obtain remote sensor data (3D acceleration, humidity, pressure, and temperature) using a web browser. In recent years, as the performances of electric devices including MEMS sensors have become well developed, we can easily use a low-power microcontroller with analog processing including an $\mathrm{A} / \mathrm{D}$ converter even with a wireless transceiver in one chip. The era is now ready for IoT sensing. For example, Fig. 11 shows a recently developed IoT device for human activity monitoring. The small and lightweight printed circuit board $\left(14 \times 14 \times 2 \mathrm{~mm}^{3}\right.$, $0.48 \mathrm{~g}$ ) possesses many types of sensors such as for 3D acceleration, humidity, atmospheric pressure, ambient temperature, and body surface temperature, and an analog frontend circuit for electrocardiogram (ECG) as well as a Bluetooth low-energy (BLE) mechanism with a chip antenna. Both Figs. 10 and 11 are IoT sensor nodes with network capability whose functions are nearly equal. However, the size, weight and power consumption are drastically reduced. This has been the evolution of microdevices in 15 years.

The system shown in Fig. 11 was fabricated as one of the works of the "Human Sensing Fusion project"(16,17) (2008-2013) supported by the Japan Science and Technology Agency (JST). The aim of this project is the technological development of small and low-power human activity monitoring systems using patch-type devices, such as an adhesive plaster. In the project, many types of work were performed, including MEMS sensors, power harvesters, flexible wiring/ substrates, unique materials such as lead zirconate titanate (PZT), polymers, and magnetic films, low-power analog/digital/RF integrated circuits, and demonstration models. In Fig. 12, although the detailed explanations are omitted, some example devices from the project are shown. ${ }^{(18)}$ The designs and fabrication processes of these devices were optimized for monolithic combo sensor devices. Other topics related to sensor networks can be found in my serial papers, entitled "Sensors in Network (1)-(5)", in Sensors and Materials. ${ }^{(19-23)}$

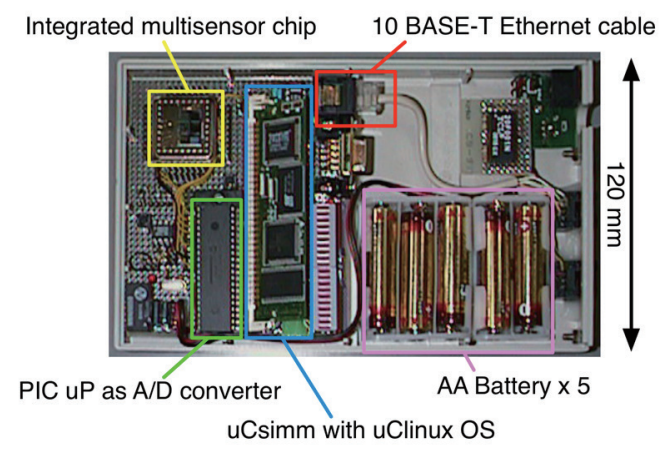

Fig. 10. (Color online) Early IoT sensor node with wired network.

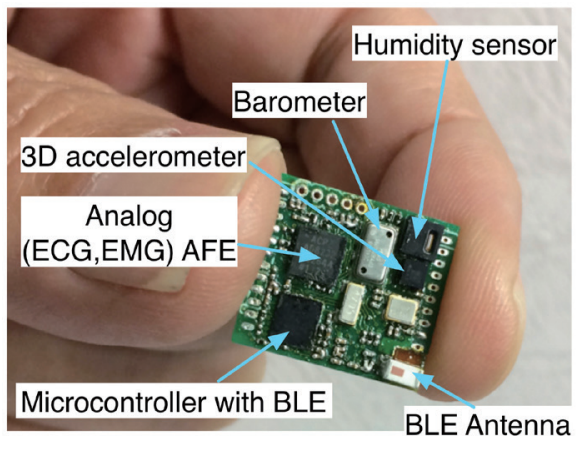

Fig. 11. (Color online) Current IoT wireless sensor node for monitoring human activity. 


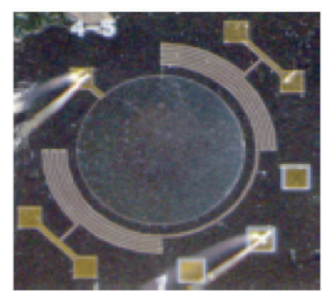

(a)

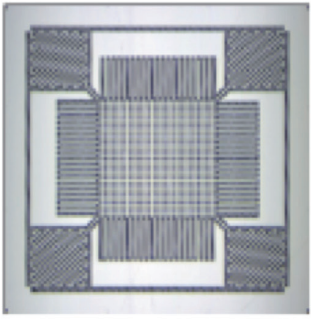

(e)

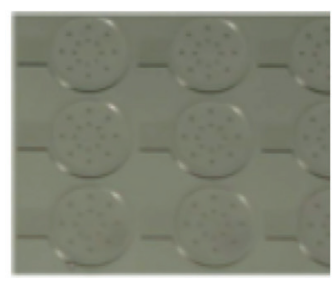

(i)

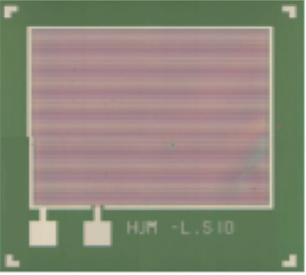

(b)

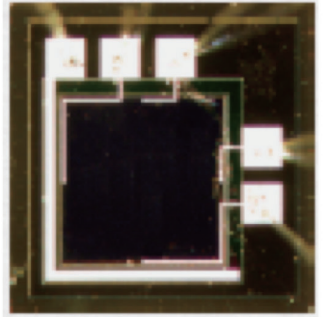

(f)

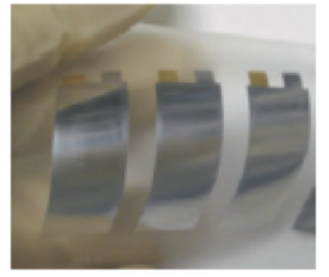

(j)

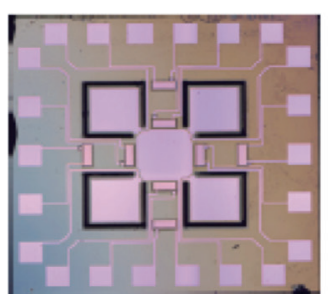

(c)

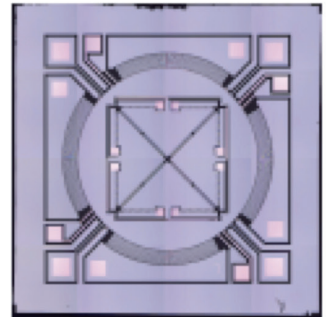

(g)

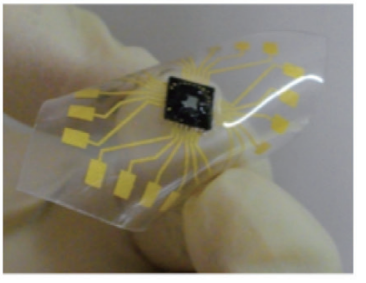

(k)

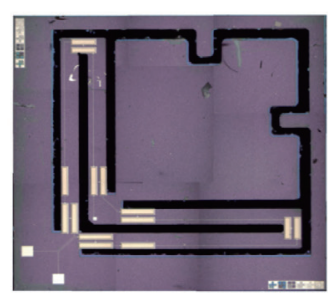

(d)

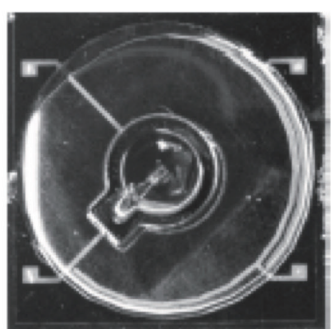

(h)

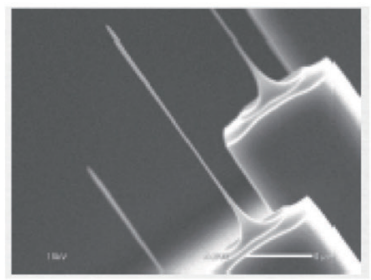

(1)

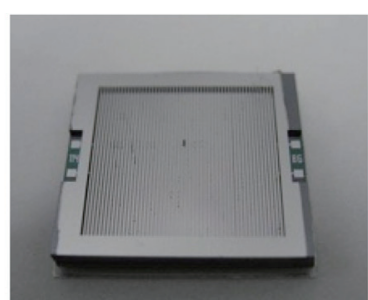

(m)

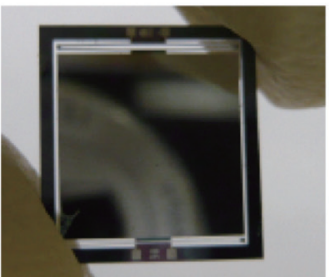

(n)

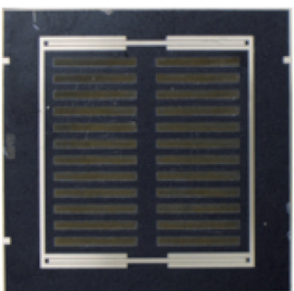

(o)

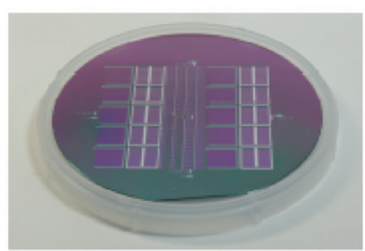

(p)

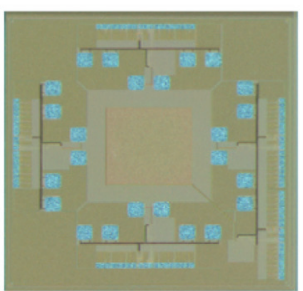

(t)

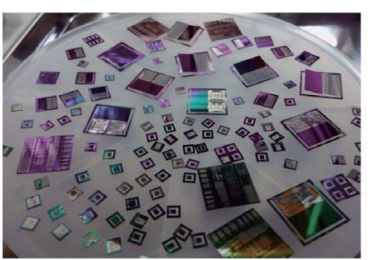

(q)

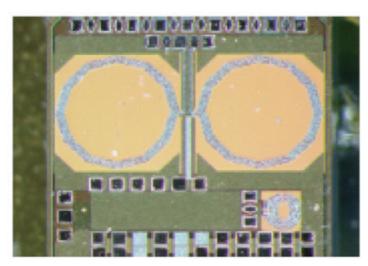

(r)

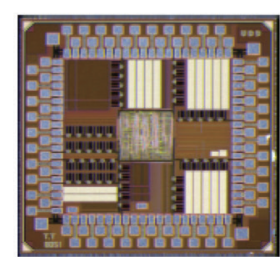

(s)

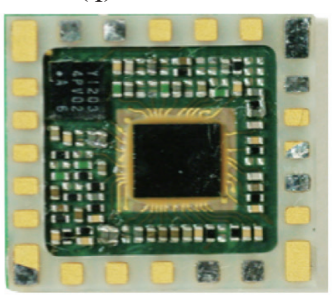

(u)

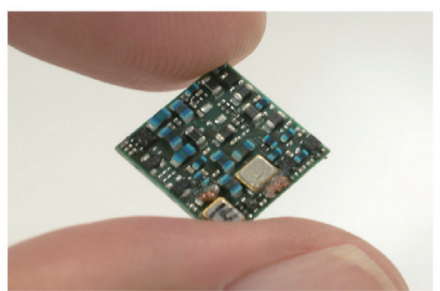

(v)

Fig. 12. (Color online) Some example devices from "Human Sensing Fusion" project: (a) barometer, (b) humidity sensor, (c) 3D shock sensor, (d) omnidirectional shock sensor, (e) accelerometer, (f) magnetic sensor, (g) gyroscope, (h) $\mathrm{SpO} 2$ sensor, (i) microphone, (j) flexible blood pulse sensor, (k) flexible wiring with sensor devices, (l) nanoneedles (painless needle), $(\mathrm{m}, \mathrm{n})$ vibratory power harvester, (o) permanent micromagnet for power harvester, (p) solar cell, (q) various PZT application devices including shock sensor and power generator, (r) RF integrated circuit, (s) lowpower microprocessor for human signal processing, (t) special sensor interface ASIC, and (u,v) $315 \mathrm{MHz}$ low-power transceiver. 


\section{Future Trends of Sensor Systems}

\subsection{One cent sensors and one-century sensors}

The current mainstream developments of MEMS sensors are the reduction in cost with miniaturization and the increase in performance. This can be said as the way to "one cent (Is it too much to say?) sensor". This tendency is very important for the coming Trillion Sensors Universe. On the other hand, different approaches for MEMS development could be considered. For example, the realization of an extremely long life sensor system for the monitoring of structures, i.e., buildings, tunnels, and bridges, is important because the structures have a lifetime in the 100-year range. Since there are many structures in the world, a maintenance-free system, throughout its lifetime, is desirable. I would like to name this as "one-century sensors". To realize such system, novel approaches are required. At first, a chemical battery or other devices using a common chemical reaction cannot be used and a practical power generator or harvester is required. Moreover, self-diagnosis, self-calibration (or cleaning etc.), self-defence, and self-healing would be crucial key technologies for one-century sensors. These functions are realized by actuators.

\subsection{Integration of actuators}

Actually, in a high-performance system, such as a human body, individual sensors have their own actuators. ${ }^{(23)}$ These actuators have various functions, such as the expansion of the dynamic range during sensing (e.g., pupil, crystalline lens), maintenance and cleaning (eyelid, lacrimal apparatus), protection (eyelid, lip), assistance of sensing functions (olfactory sense with airflow by respiration; tactile sense for detecting shear stress by moving fingers on an object), and the addition of new functions (e.g., specifying the direction of a sound source by turning one's head). The one-century sensor system should have such actuators for maintaining its functionalities. Of course, actuators consume additional energy, so the importance of duty of a power source will increase further in the one-century sensor system.

\section{Conclusions}

The progress of sensor intelligence was described. Recently, extremely rapid progress has been made in the MEMS sensor field and the dreams of the old days have become true one after another. For the coming Trillion Sensors Universe, we should have new dreams and desires and continuously work towards the next generation of MEMS.

\section{References}

1 K. E. Petersen: Proc. IEEE 70 (1982) 420.

2 S. Middelhoek, J. B. Angell, and D. J. W. Noorlag: IEEE Spectrum 17 (1980) 42.

3 R. A. Breckenridge and C. Husson: Proc. AIAA/ NASA Conf. on Smart Sensors, Hampton, (1978) https://arc. aiaa.org/doi/10.2514/6.1978-1722. 
4 E. S. Sinencio and A. G. Andreou Ed.: Low-voltage/Low-power Integrated Circuits and Systems (IEEE, Inc., New York, 1999).

5 M. Nakamura, K. Murakami, H. Nojiri, and T. Tominaga: Int. Conf. Solid-State Sensors and Actuators (Transducers 1987) pp. 112-115.

6 S. Sugiyama, M. Takigawa, and I. Igarashi: Sens. Actuators 4 (1982) 113.

7 A. McNeil: Improving the MEMS Pressure Sensor, Sensors OnLine, https://www.sensorsmag.com/ components/improving-mems-pressure-sensor (accessed July 2000)..

8 K. Maenaka, T. Ohgusu, M. Ishida, anXd T. Nakamura: Int. Conf. Solid-State Sensors and Actuators (Transducers 1987) 523-526.

9 K. Maenaka, M. Tsukahara, and T. Nakamura: Sens. Actuators, A 22 (1990) 747.

10 K. Maenaka, H. Okada, and T. Nakamura: Sens. Actuators, A 22 (1990) 807.

11 Analog Devices: Analog Dialogue 30 (1996) 3.

12 K. Maenaka and T. Shiozawa: Int. Conf. Solid-State Sensors and Actuators (Transducers 1993) 642-645.

13 J. A. Green, S. J. Sherman, J. F. Chang, and S. R. Lewis: IEEE J. Solid-State Circuits 37 (2002) 1860.

14 F. Larmer and A. Schilp: German Patent DE4241045C1.

15 T. Fujita, K. Inoue, A. Tsuchitani, S. Arita, and K. Maenaka: Int. Conf. Solid-State Sensors and Actuators (Transducers 2001) 88-91.

16 K. Maenaka: IEEE Int. Conf. Nanotechnology (IEEE-NANO2016) Th2-2.

17 H. Eren and J. G. Webster Ed.: Telemedicine and Electronic Medicine: Development of Disposable Adhesive Wearable Human-Monitoring System (CRC press, Boca Raton, 2015) p. 301.

18 K. Maenaka: IEEJ Trans. Sens. Micromach. 134 (2014) 372 (In Japanese).

19 K. Maenaka: Sens. Mater. 28 (2016) 745.

20 K. Maenaka: Sens. Mater. 28 (2016) 927.

21 K. Maenaka: Sens. Mater. 28 (2016) 1077.

22 K. Maenaka: Sens. Mater. 28 (2016) 1149.

23 K. Maenaka: Sens. Mater. 28 (2016) 1247.

24 J. Nakamura, K. Higuchi, and K. Maenaka: Microsyst. Technol. 19 (2013) 433. 